\title{
PERSPECTIVAS PARA 0 GERENCIAMENTO AMBIENTAL DA BAÍA DO ALMIRANTADO, ILHA REI GEORGE, ANTÁRTICA*
}

\author{
Jorge Arigony Neto**, Jefferson Cardia Simões***, Ulisses Franz Bremer*** e Norberto Dani ***
}

\begin{abstract}
Resumo: Este trabalho descreve o uso inicial de um sistema de informações geográficas (SIG) para auxiliar no gerenciamento da Área Antártica Especialmente Gerenciada (AAEG) da baía do Almirantado, exemplificado mediante os resultados preliminares obtidos. Seguindo recomendações do Protocolo ao Tratado Antártico sobre Proteção ao Meio Ambiente (Protocolo de Madrid), foi utilizada informação geoespacial integrada em SIG para demarcar os limites da AAEG. A área foi recalculada em $362 \mathrm{~km}^{2}$ (antes fora estimada em $370 \mathrm{~km}{ }^{2}$ ). Com base na sobreposição de informações na península Keller (no norte da baía do Almirantado), foi representado espacialmente o local onde, seguindo as definições do Protocolo de Madrid, havia sido detectado impacto ambiental menor ou transitório no trabalho de campo. A possibilidade de utilização do SIG recém-implantado no gerenciamento ambiental da baía do Almirantado cresce à medida que surge a necessidade de escolha de áreas aptas à ocupação humana, bem como da avaliação do impacto ambiental causado pelas atividades na área.
\end{abstract}

Palavras-chave: Antártica, Ilha Rei George, Gerenciamento ambiental, SIG.

\section{Introdução}

A década de 1990 foi marcada pelo aumento da atividade turística na Antártica (Fig. 1) (IAATO, 2002). Distante cerca de $1.000 \mathrm{~km}$ do extremo sul da América do Sul, a península Antártica (Fig. 2) e os arquipélagos adjacentes servem de destino a $90 \%$ dos turistas que se dirigem ao sul do paralelo $60^{\circ} \mathrm{S}$. Em função de sua beleza paisagística, abundante vida selvagem, diversas enseadas e baías protegidas para ancoragem, as ilhas Shetland do Sul (Fig. 2) recebem, todos os anos, a maioria desses turistas, que nelas chegam após dois a três dias de viagem maritima. Essa proximidade com o continente sul-americano tem também favorecido o estabelecimento de grande número de estações científicas, meteorológicas, refúgios e 0 abrigo temporário de grupos de pesquisa no arquipélago, o que é facilitado pelo transporte aéreo em viagens de duas horas e meia desde

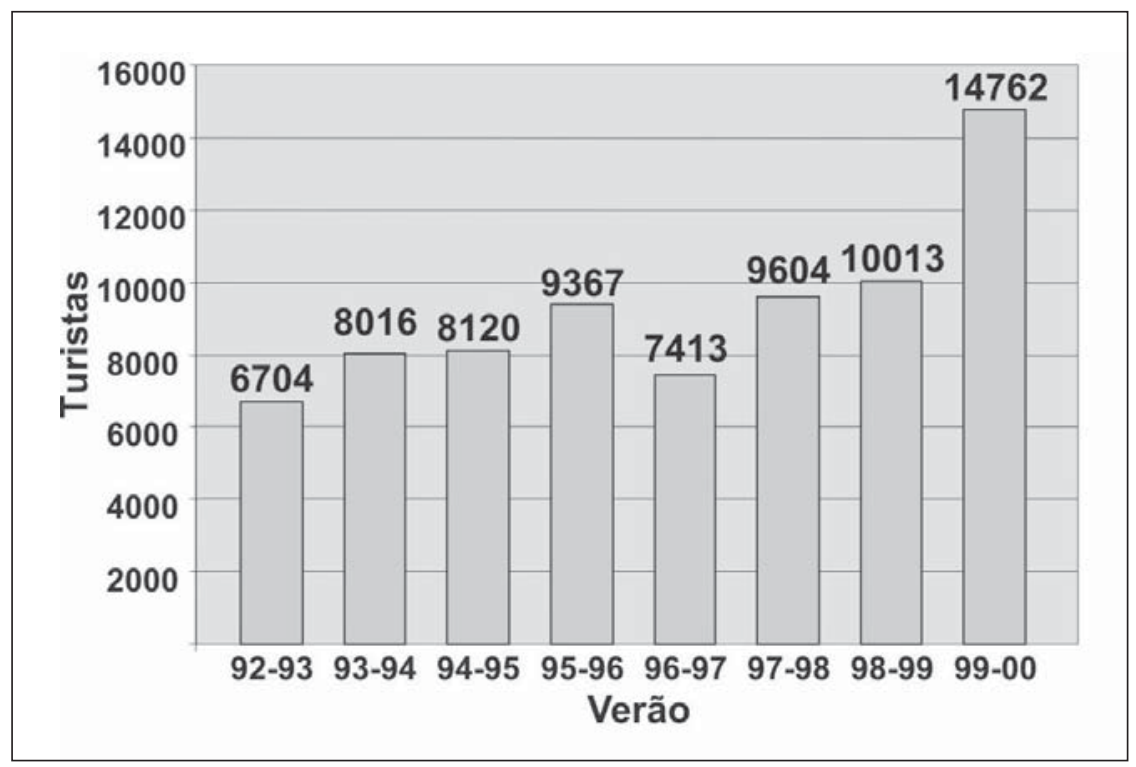

Figura 1 Gráfico de visitação turistica na Antártica entre 1992/1993 e 1999/2000. Fonte: IAATO ( 2002).

Punta Arenas, no Chile, ou de duas horas desde Ushuaia, na Argentina, até a pista de pouso da ilha Rei George (IRG) (Fig. 2).

* Pesquisa subvencionada pelo CNPq processo 48.0243/00-0).

** Institut für Physische Geographie Albert-Ludwigs-Universität Freiburg, Werderring 4 - D-79085 Freiburg, Germany. e-mail:

jorge.arigony@geographie.uni-freiburg.de

*** Laboratório de Pesquisas Antárticas e Glaciológicas (LAPAG), Universidade Federal do Rio Grande do Sul, Av. Bento Gonçalves 9500, Bairro Agronomia, C.P., 150001, 91501-970 Porto Alegre (RS),Brasil. e-mail: jefferson.simoes@ufrgs.br-bremer@ufrgs.br

**** Departamento de Geodésia, Universidade Federal do Rio Grande do Sul, Av. Bento Gonçalves 9500, Bairro Agronomia, C.P., 150001, $91501-970$ Porto Alegre (RS), Brasil. e-mail: norberto.dani@ufrgs.br 


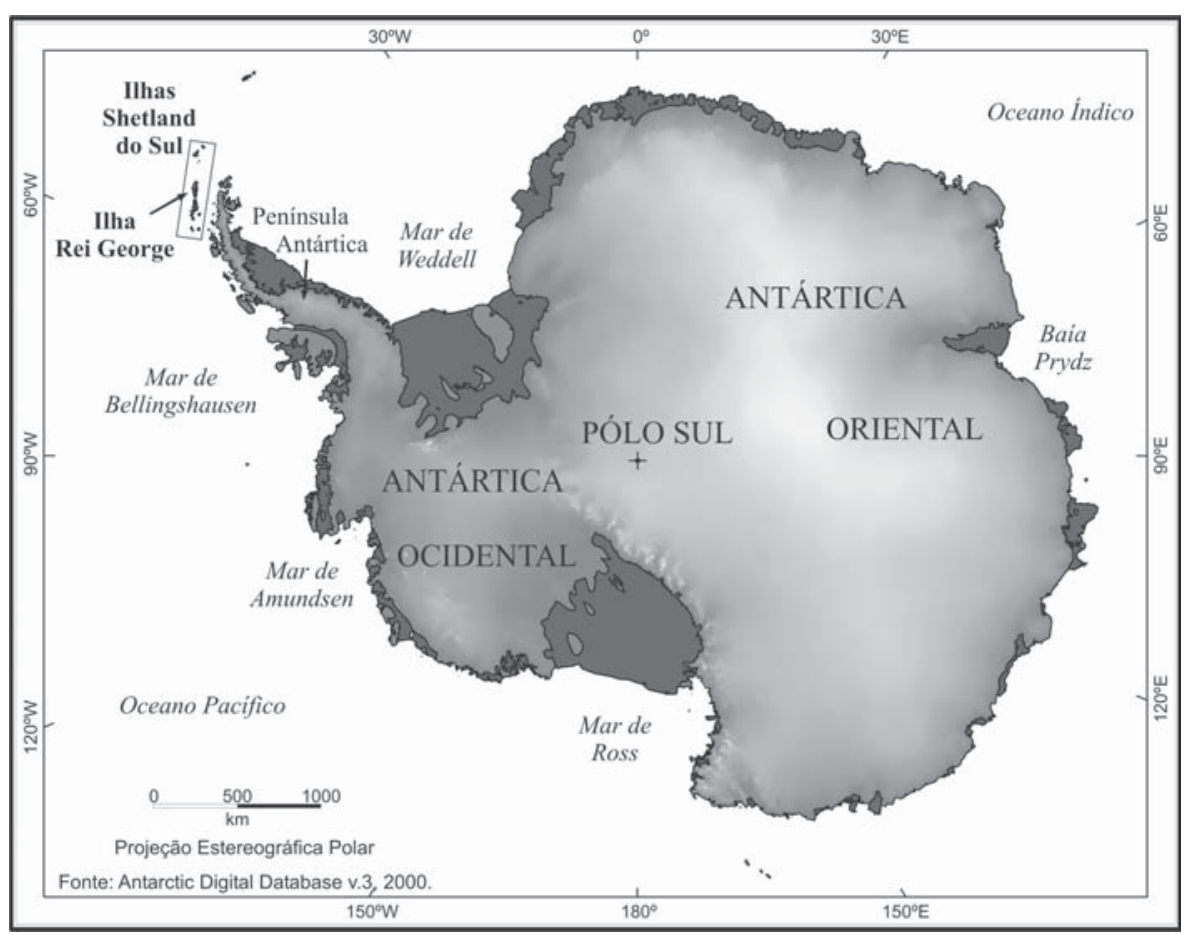

Figura 2 Localização da ilha Rei George. Fonte: Antarctic Digital Database v.3 (BAS, 2000). ambientais em campos de musgos e locais de nidificação de aves, causados pela presença de pesquisadores que permanecem nas estações científicas e refúgios, e por turistas que anualmente visitam as praias da região.

0 Sistema do Tratado Antártico tem gerado mecanismos para promover o gerenciamento ambiental e das atividades humanas na Antártica (e.g., Protocolo de Madri, ATCPs, 1993). Baseados nesse protocolo e com o objetivo de evitar e minimizar o risco de impactos cumulativos no meio ambiente, intensificar a assistência e a cooperação entre os países que operam na baía do Almirantado e proteger importantes características ambientais e históricas, Brasil e Polônia propuseram a criação da primeira Área Antártica Especialmente Gerenciada (AAEG), elaborando um Plano de Gerenciamento para a mesma (ATCPs, 1996).
Impactos decorrentes da presença humana na Antártica têm sido registrados por diversos autores (GREENPEACE, 1990; HARRIS, 1991; HANSON \& GORDON, 1998). Na IRG, particularmente, a atividade humana tem gerado considerável impacto ao ecossistema local (HARRIS, 1991). Além dos turistas, na ilha são realizadas atividades de pesquisadores distribuídos em nove estações científicas permanentes e diversos refúgios e estações de verão. Somente na área da baía do Almirantado existem três estações científicas (duas que operam durante o ano inteiro) e seis refúgios, com uma população estimada em 100 pessoas durante o verão austral e 20 no inverno, além dos visitantes que, no verão de 1999/2000, por exemplo, totalizaram 2.327 pessoas (IAATO, 2002). Na baía do Almirantado (Fig. 3), HARRIS (1991) descreveu os impactos gelo da Ilha Rei George.

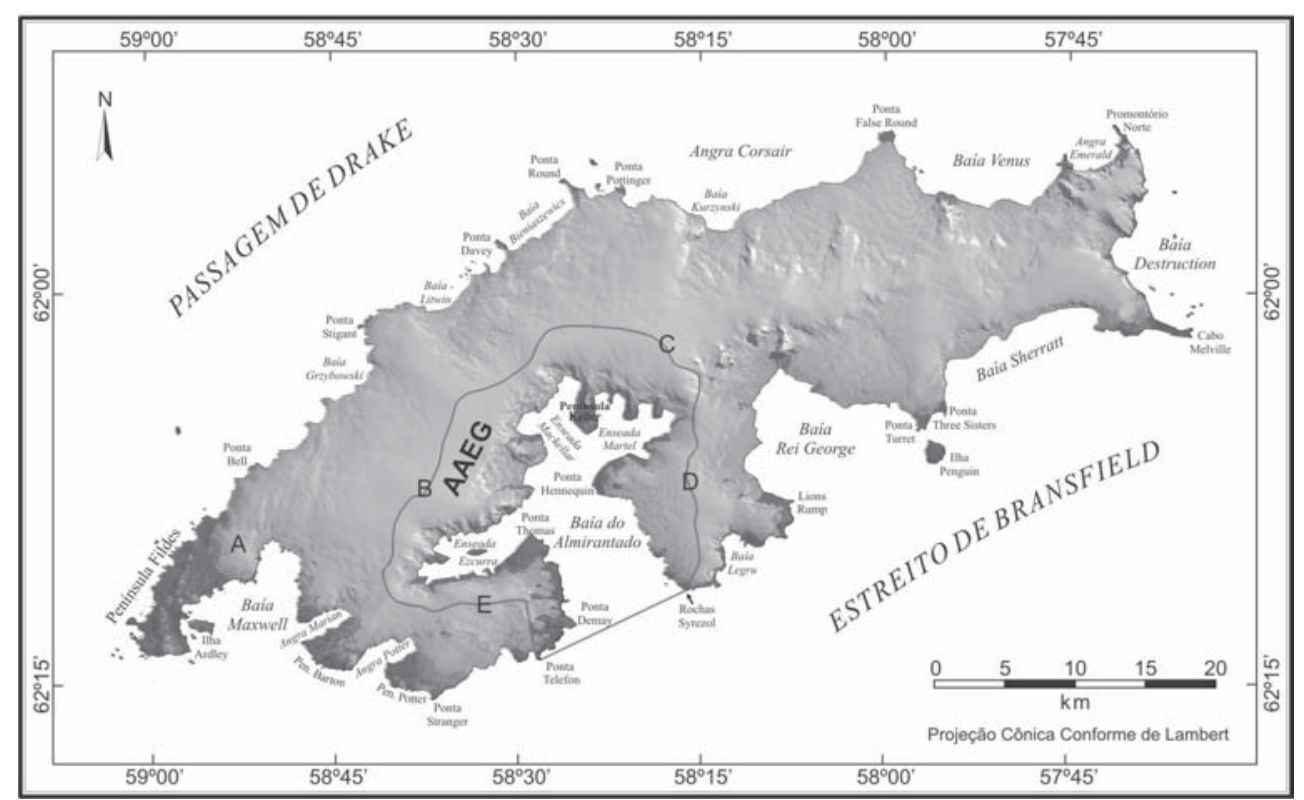

Figura 3 Nova delimitação da AAEG Baía do Almirantado. As letras A-E marcam os principais domos da calota de 
caracteristicas de múltipla utilização (científica, logística e turistica), SIMÕES et al. (2001) propuseram a utilização de um sistema de informações geográficas (SIG) para administrar os conflitos de uso e monitorar as mudanças ambientais e o impacto humano na área, servindo de apoio ao Plano de Gerenciamento da AAEG.

Com a implementação do SIG para a AAEG Baía do Almirantado (ARIGONY NETO et al., no prelo), este trabalho descreve os primeiros passos em direção à utilização desse sistema para o gerenciamento ambiental na área, os quais são exemplificados por meio dos resultados preliminares obtidos.

\section{Materiais e métodos}

\section{Materiais}

No trabalho foram utilizados dados de diversas fontes (e.g., dados de sensoriamento remoto; levantamento de campo realizado com GPS - Global Positioning System; modelo digital de elevação - MDE), integrados ao SIG da AAEG baía do Almirantado. Na Tab. 1 são apresentadas as principais características desses dados, relacionando as informações que foram obtidas e integradas ao SIG.

Durante a realização do trabalho de campo, utilizou-se um par de receptores GPS Magellan ProMARK X-CM. Para a integração das informações foi utilizado o ArcGIS (Environmental Systems Research Institute - ESRI), prestando-se plenamente para a compatibilização dos dados.

\section{Métodos}

Realizada a definição da base cartográfica, do programa para integração dos dados (ArcGIS) e da forma de representação da informação espacial (ARIGONY NETO et al., no prelo), novos planos de informação foram criados com dados préexistentes e com outros obtidos em campo. Abaixo segue uma breve descrição desse trabalho.

Tendo como base os trabalhos desenvolvidos por BREMER (1998) e SIMÕES et al. (1999), as bacias de drenagem glacial foram delimitadas, com a utilização do ArcGIS, sobre o mosaico de imagens SPOT com as curvas de nivel sobrepostas.

A utilização de curvas de nível aumentou a precisão na delimitação das bacias de drenagem realizada anteriormente por BREMER (1998). As curvas de nivel demarcaram mais precisamente os limites em que as mudanças de declividade são suaves e não permitem a precisa distinção em imagens de satélite (e.g., limite noroeste da geleira Domeyko) (Fig. 4).

Tabela 1 Dados utilizados na realização do trabalho. Modificado de ARIGONY NETO et al. (no prelo).

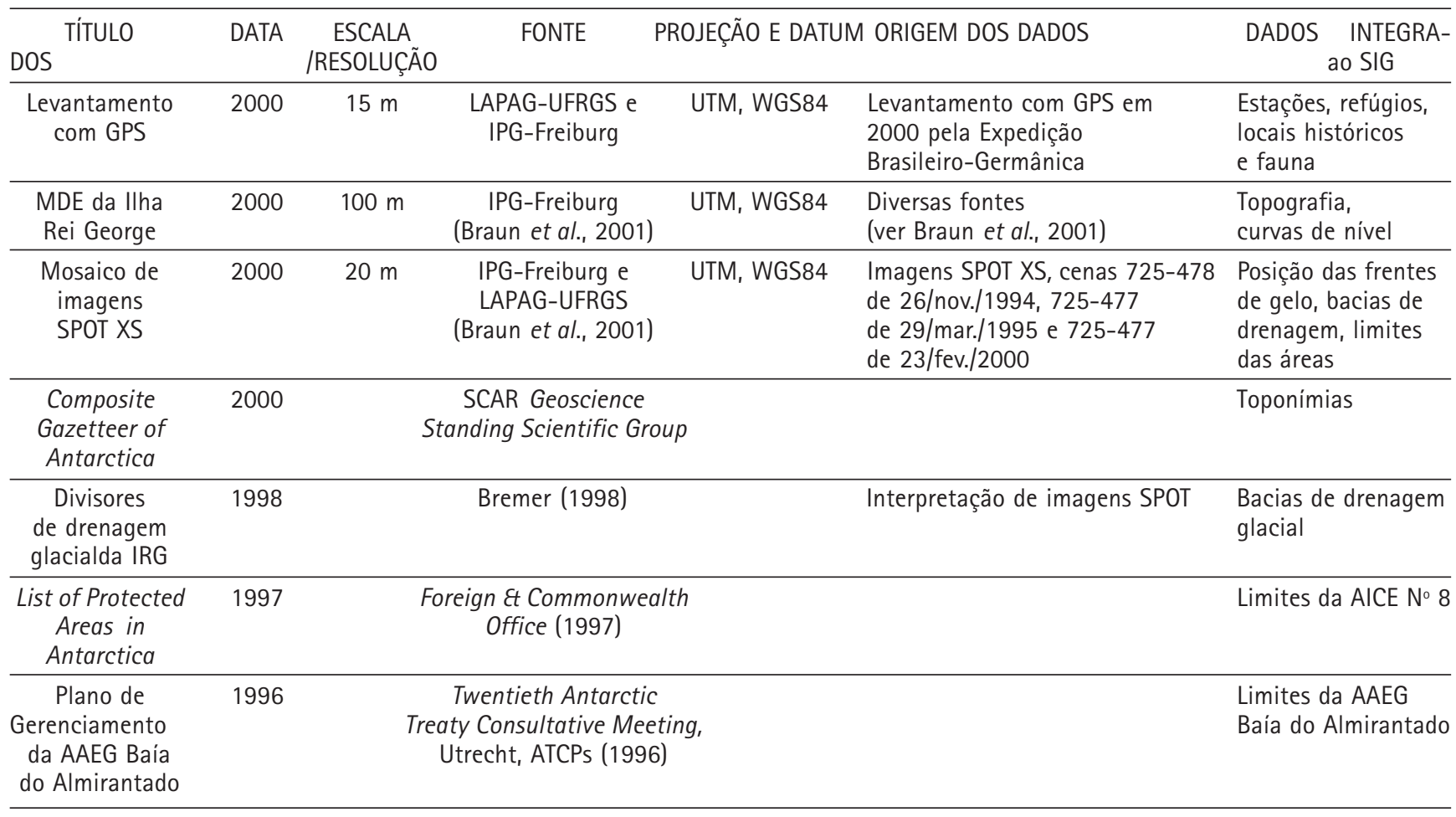




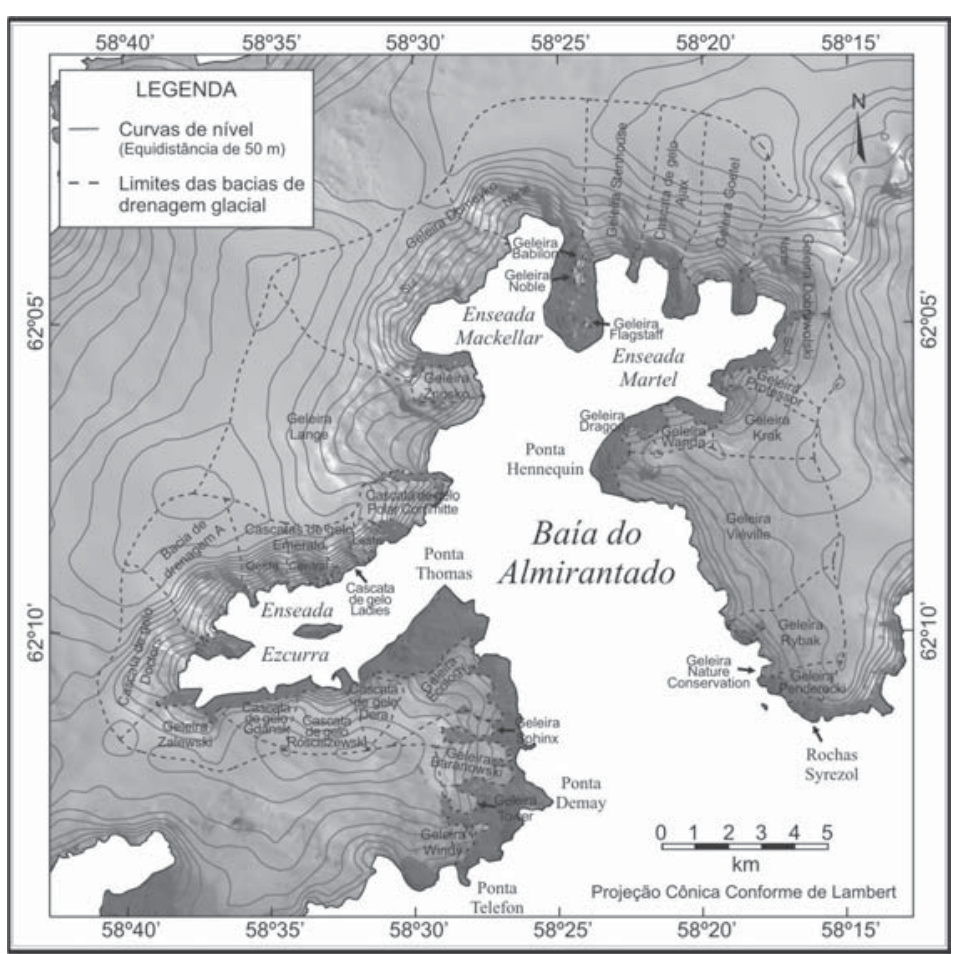

Figura 4 Delimitação das bacias de drenagem das geleiras da AAEG.

Estações científicas e refúgios na península Keller, no norte da AAEG (Figs. 4 e 5), foram mapeados por levantamento de campo realizado com a utilização do GPS. As coordenadas de cada feição foram obtidas com base no cálculo da média aritmética de 1.500 observações coletadas por medida, o que esteve associado a um erro médio quadrático (RMS) de aproximadamente 15 m (MAGELLAN CORPORATION, 1999).

Os principais caminhos percorridos por turistas e pesquisadores na península Keller foram digitalizados diretamente sobre o mosaico de imagens SPOT, tendo como base os mapas do plano de gerenciamento da AAEG Baía do Almirantado (ATCPs, 1996). Feições como a EACF, os refúgios e a linha de costa serviram de apoio para a localização dos caminhos.

A delimitação da Área de Interesse Científico Especial № $8^{1}$ (AICE № 8), ocorreu mediante a digitalização sobre o mosaico SPOT, utilizando a descrição dos limites da área (FOREIGN \&t COMMONWEALTH OFFICE, 1997). Os limites da AAEG Baía do Almirantado foram baseados na descrição da área apresentada no plano de gerenciamento (ATCPs, 1996). A nova delimitação das bacias de drenagem glacial da baía do Almirantado permitiu aperfeiçoar os limites. Esse plano de informação foi inserido com a digitalização do limite da AAEG no estreito de Bransfield e a sobreposição dos divisores da bacia de drenagem da baía do Almirantado e dos limites da AICE № 8.

Duas colônias de gaivotões (Larus dominicanus) e um campo de nidificação de skuas (Catharacta sp.), localizados na península Keller, formam o primeiro plano de informações referente à biologia da AAEG. Essas colônias foram escolhidas por estarem localizadas na mesma península onde se encontra instalada a EACF, estando, então, suscetiveis à interferência humana devido à falta de obstáculos naturais (e.g., geleiras) que as separem da estação. As coordenadas de localização dessas colônias foram obtidas no levantamento de campo, com a utilização de GPS.

Com a integração das informações descritas acima, foram utilizados métodos básicos de visualização de informação espacial em SIG, tais como: sobreposição de planos de informação e visualização tridimensional. A visualização da informação ambiental intregrada sobre uma mesma base geo-espacial permitiu a realização de uma revisão descritiva das características geográficas, o que é apresentado na seção seguinte.

\section{Resultados e discussões}

\section{Revisão dos limites e principais características geográficas da área}

De acordo com o Anexo $V$ do Protocolo de Madri (ATCPs, 1993), os planos de gerenciamento para áreas protegidas na Antártica devem incluir mapas e fotografias que mostrem claramente os limites da área em relação a feições de destaque.

A AAEG Baía do Almirantado 2 foi delimitada inicialmente de forma analógica sobre carta topográfica do Antarctic Place Names Committee (British Foreign and Commonwealth Office), na escala 1: 100.000 (ATCPs, 1996). Com a utilização do mosaico de imagens de satélite e curvas de nivel, foi realizada uma nova delimitação da AAEG (Fig.3).

1 Regulamentada pelo Anexo V do Protocolo de Madrid (ATCPs, 1993), a Área de Interesse Científico Especial (AICE) é criada para proteger valores ambientais, cientificos, históricos, estéticos e selvagens. Permissões são necessárias para o ingresso nessa área. A AICE № 8, particularmente, tem como objetivo proteger pesquisa ornitológica de longa duração.

2 A Área Antártica Especialmente Gerenciada Baía do Almirantado compreende a região considerada como imediatamente dentro da bacia de drenagem glacial da baia de mesmo nome, incluindo a porção da Área de Interesse Científico Especial № 8 (AICE № 8), que se localiza fora da bacia de drenagem (Foreign \& Commonwealth Office, 1997). 
A sua área foi recalculada em $362 \mathrm{~km}^{2}$ (anteriormente estimada em $370 \mathrm{~km}^{2}$ ), sendo $194 \mathrm{~km}^{2}$ cobertos pela calota de gelo da IRG e pequenas geleiras de anfiteatro e $30 \mathrm{~km}^{2}$ de áreas livres de gelo. $0 \mathrm{~s} 138 \mathrm{~km}^{2}$ restantes correspondem a águas da baía do Almirantado $\left(131 \mathrm{~km}^{2}\right)$ e do estreito de Bransfield $\left(7 \mathrm{~km}^{2}\right)$. A Tab. 2 estabelece a comparação entre os valores apresentados no plano de gerenciamento e aqueles calculados neste trabalho.

As diferenças entre os valores apresentados na Tab. 2 refletem, em geral, as variações glaciais ocorridas na área entre 1956 e 2000 (ARIGONY NETO et al., 2001). Por outro lado, as diferenças encontradas nos valores das áreas livres de gelo representam estimativas equivocadas geradas pela falta de dados cartográficos ou satelitais precisos durante a primeira delimitação da área.

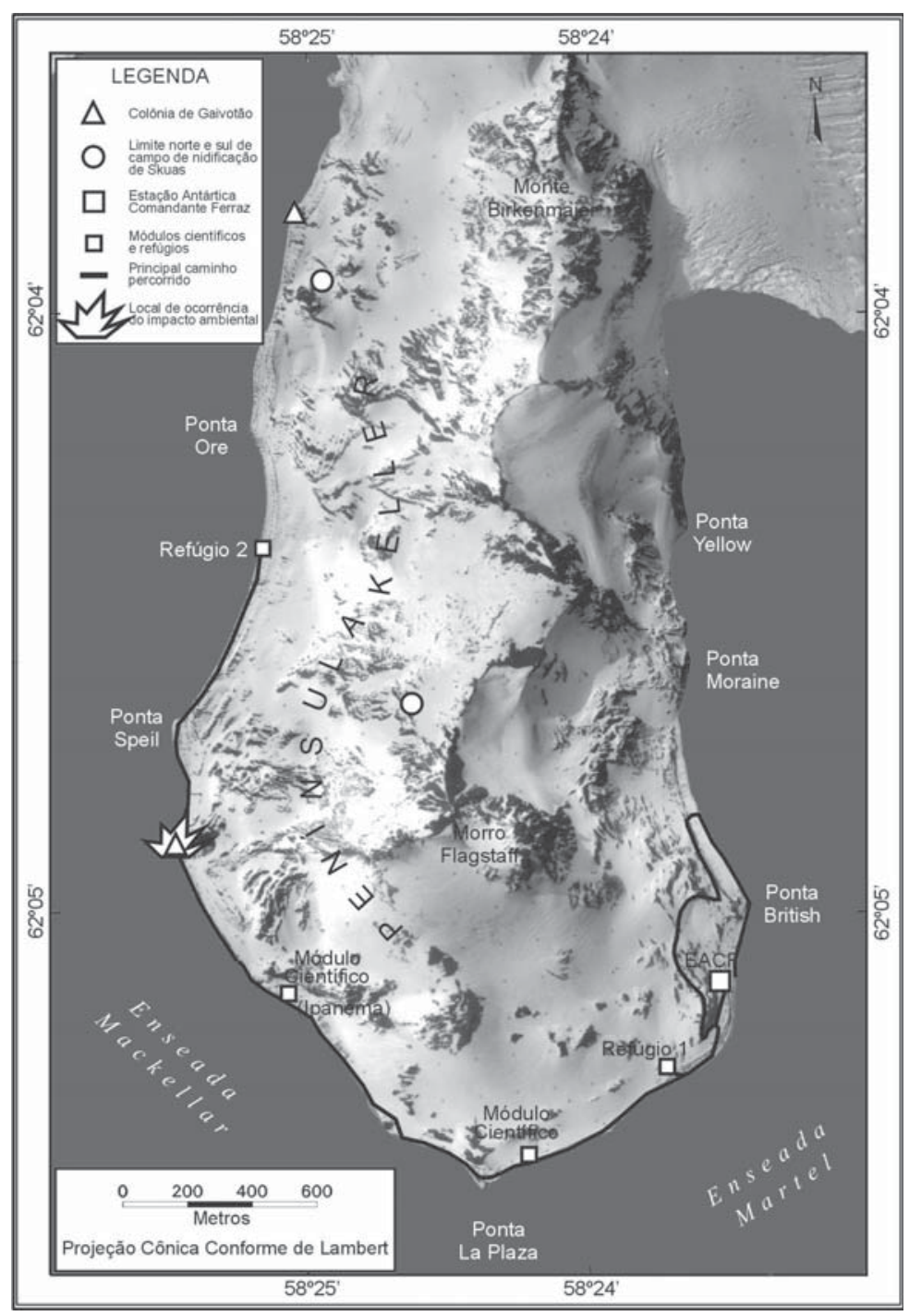

Tabela 2 Valores de áreas $\left(\mathrm{km}^{2}\right)$ da AAEG Baía do Almirantado apresentados no plano de gerenciamento e calculados no SIG da AAEG.

\begin{tabular}{lcc}
\hline Áreas AAEG & Plano de Gerenciamento & SIG AAEG \\
\hline Áreas cobertas por gelo & 203 & 194 \\
Áreas livres de gelo & 37 & 30 \\
Baía do Almirantado & 122 & 131 \\
Estreito de Bransfield & 8 & 7 \\
\hline Área Total & 370 & 362 \\
\hline
\end{tabular}

Representando aproximadamente 53,6\% da AAEG, a calota de gelo da IRG possui dois de seus três principais domos de gelo, B e C na Fig. 3, delimitando essa área a norte e a noroeste. Esses domos estão conectados um ao outro e ao terceiro (domo A) na principal direção da ilha, SW-NE (SIMÕES et al., 1999). A maior altitude é encontrada no domo $C_{1}$ com $706 \mathrm{~m}$.

A leste e a sudoeste, a AAEG tem como limite os pequenos platôs dos campos de gelo que levam 0 nome das penínsulas onde estão localizados. Na Fig. 3, esses platôs estão representados pelas letras $D$ (campo de gelo Kraków) e E (campo de gelo Warsawa).

No setor da baía do Almirantado, a calota de gelo da ilha apresenta várias geleiras de descarga que drenam o platô através de depressões bem definidas, como a geleira Lange (Fig. 4), com rápido fluxo e muitas vezes terminando em cascatas de gelo (e.g., geleiras Stenhouse, Ajax e Polar Committee). A Fig. 4 apresenta as 35 bacias de drenagem glacial que estão dentro da AAEG. Desse total, 32 drenam o campo de gelo, as restantes são pequenas geleiras de anfiteatro da península Keller (geleiras Flagstaff, Noble e Babilon).

Os $30 \mathrm{~km}^{2}$ de áreas livres de gelo (8,3\% da AAEG) são formados por "oásis" e nunataks (RAKUSASUSZCZEWSKI et al., 1993). Segundo MARSZ \& RAKUSA-SUSZCZEWSKI (1987) apud RAKUSA-SUSZCZEWSKI et al. (1993), o termo "oásis" refere-se a áreas de terra separadas por massas de gelo de diferentes dimensões. Por causa da sua localização costeira na baía do Almirantado, esses "oásis" abrigam a maioria das espécies animais e vegetais terrestres do local.

Figura 5 Localização das colônias na península Keller, EACF, módulos, refúgios, caminhos utilizados por pesquisadores e local do impacto ambiental detectado. Fotografia aérea de $26 / 01 / 1975$. Seta localiza o esqueleto de baleia montado por Jacques Cousteau. 
Ocupação humana permanente e turismo: conflitos com áreas de reprodução da fauna e flora na península Keller.

A península Keller foi escolhida para este estudo por abrigar a Estação Antártica Comandante Ferraz (EACF) e dois refúgios distribuídos nos dois lados da península (Fig. 5), o que garante uma movimentação humana permanente, sendo bastante acentuada no verão. A população fixa é estimada em 44 pessoas no período de verão (de dezembro a março) e 13 pessoas no inverno. Turistas desembarcam em suas praias todos os anos, sendo que, no verão de 1999/2000, 387 deles visitaram a península Keller, sem considerar as pessoas que chegam nos navios de apoio logístico (tripulação e pessoal da manutenção das estações).

Impactos ambientais relacionados a atividades da EACF foram apresentados pelo GREENPEACE (1990), que, durante expedição à Antártica em 1989, visitou a estação brasileira e observou a possivel existência de distúrbios em ninhos de trinta-réis do Ártico (Sterna vittata) nas suas proximidades. HARRIS (1991) observou que os mesmos ninhos sofrem interferências de pesquisadores curiosos e turistas. $E_{1}$ no nosso trabalho de campo, foi verificado que o campo de musgos situado ao redor de um esqueleto de baleia montado pelo explorador francês Jacques Cousteau (Fig. 5) apresenta marcas de rodas ocasionadas provavelmente por quadriciclos.

Para a definição de impacto ambiental, foi utilizado o conceito do Grupo de Avaliação Ambiental (GAAm) do Programa Antártico Brasileiro (PROANTAR, 2000), o qual baseia-se na Resolução 01/86 do Conselho Nacional do Meio Ambiente - Conama (Brasil, 2001):

Segundo essa definição, um impacto ambiental corresponde a quaisquer alterações das propriedades físicas, químicas ou biológicas do meio ambiente, causadas por qualquer forma de matéria ou energia resultante de atividades que, direta ou indiretamente afetem: a) a saúde, a segurança e o bem-estar da população; b) as atividades sociais e econômicas; c) a biota; d) as condições estéticas e sanitárias do meio ambiente; e e) a qualidade dos recursos naturais (CONAMA, 2001).

Com relação a gradações do impacto ambiental, são utilizados os níveis propostos pelo Protocolo de Madri (ATCPs, 1993):

a) um impacto menor ou transitório; b) superior a um impacto menor ou transitório; e c) inferior a um impacto menor ou transitório. Em função do protocolo não definir o que corresponde a "um impacto menor ou transitório", são novamente utilizadas as definições do GAAm (PROANTAR, 2000):
Segundo estas definições: a) Será considerado impacto menor ou transitório aquele que for observável durante apenas um curto período de tempo, que não ultrapasse a capacidade assimiladora natural local de seus efeitos e que, em particular, não introduza espécies exóticas na região, modifique de forma claramente visivel os habitats naturais e turísticos, a fisiografia local, os valores estéticos, históricos, naturais e turisticos, introduza contaminantes em niveis superiores aos padrões internacionais, gere ruidos e odores que possam perturbar o comportamento normal das espécies da região, perturbe outras atividades e a segurança humana e que, conseqüentemente, ainda que possa requerer algumas medidas de mitigação, não exija medidas de recuperação, nem de avaliação de alternativas locacionais e tecnológicas.

b) Será considerado impacto superior a um impacto menor ou transitório todo aquele cujos efeitos sejam de curta ou longa duração, que ultrapasse a capacidade assimiladora natural local de seus efeitos, que viole acordos internacionais sobre a proteção dos ecossistemas antárticos e de seus recursos naturais, bem como provoque riscos à segurança humana, e que, em particular, reduza a diversidade específica, e que, conseqüentemente, requeira medidas efetivas de mitigação/eliminação, através inclusive, da avaliação de alternativas locacionais e tecnológicas, bem como de recuperação de ambientes atingidos.

c) Será considerado inferior a um impacto menor ou transitório aquele que seja de muito curta duração, que não induza qualquer efeito mencionado no primeiro item, em qualquer grau, nivel e extensão, intensidade etc., que não requeira qualquer medida de mitigação, e, em especial, que possa ser repetido sem atingir o limite da capacidade assimiladora de seus efeitos, e que, estritamente, caracterize-se como uma pequena atividade resultante ou necessária a uma pesquisa científica/tecnológica, bem como as que forem ser realizadas em estações permanentes ou estacionais de pesquisa (PROANTAR, 2000).

Durante a execução do trabalho de campo foi identificado um local onde está ocorrendo impacto ambiental. A época de maior visitação turística e maior número de pesquisadores na EACF, embora restrita a poucos meses por ano (verão), coincide com o período de reprodução das aves. 0 trecho do caminho localizado entre o módulo Ipanema e o Refúgio 2, que, na maioria das ocasiões, é percorrido com veículo a motor (quadriciclo), passa exatamente por uma colônia de gaivotões e próximo do setor sudoeste do campo de nidificação de skuas (Fig. 5), 
gerando ruídos que perturbam o comportamento normal das aves adultas, as quais deixam os filhotes desprotegidos e mais vulneráveis à ação dos predadores. Seguindo as definições de impacto ambiental propostas pelo GAAm (PROANTAR, 2000) descritas acima, podemos considerar que esteja ocorrendo impacto menor ou transitório, pois a perturbação é observável durante apenas um curto de período de tempo (verão).

0 SIG da AAEG foi utilizado para a representação espacial da área impactada. Essa tarefa foi realizada pela sobreposição dos planos de informação contendo as duas colônias de gaivotões, o campo de nidificação de skuas e o principal caminho percorrido na península por pesquisadores, pessoal de apoio da EACF e do navio de apoio oceanográfico Ary Rongel (Fig. 5).

Como medida mitigadora ao impacto e na falta de dados a respeito do comportamento animal (e.g., tolerância a aproximações), sugere-se simplesmente que o trânsito nesse local seja restrito a deslocamento a pé, evitando a utilização de quadriciclos. Para o transporte de equipamento ou material a ser utilizado no Refúgio 2 e em áreas vizinhas, sugere-se 0 deslocamento por via marítima. $E_{1}$ às pessoas que necessitem percorrer a costa oeste da península Keller, sugere-se que contornem os pequenos promontórios colonizados por grupos de gaivotões, evitando assim o pisoteio de ovos e pequenos filhotes, bem como diminuindo as perturbações que expõem estes últimos às skuas que nidificam nas proximidades.

\section{Conclusões}

Novos dados e interpretações de levantamentos prévios foram obtidos para a AAEG, sendo resumidos nos parágrafos seguintes:

1) os limites da AAEG foram revistos e sua área foi recalculada, obtendo-se o valor de $362 \mathrm{~km}^{2}$ (anteriormente, a área foi estimada em $370 \mathrm{~km}^{2}$ ), sendo $194 \mathrm{~km}^{2}$ cobertos pela calota de gelo da IRG e pequenas geleiras de anfiteatro e 30 $\mathrm{km}^{2}$ de áreas livres de gelo. $0 \mathrm{~s} 138 \mathrm{~km}^{2}$ restantes correspondem às águas da baía do Almirantado $\left(131 \mathrm{~km}^{2}\right)$ e do estreito de Bransfield $\left(7 \mathrm{~km}^{2}\right)$.
Apesar desses valores não representarem grandes diferenças, temos a vantagem de dispor de dados digitais que podem ser integrados facilmente a outros planos de informação e utilizados com maior eficácia para o gerenciamento da área.

2) A identificação de conflitos entre ocupação humana e as áreas de reprodução animal, realizada na península Keller, levou-nos à constatação de impacto ambiental menor ou transitório, segundo as definições do Protocolo de Madri (ATCPs, 1993) e GAAm (PROANTAR, 2000). Sugere-se, então, simplesmente o deslocamento a pé entre o módulo Ipanema e o Refúgio 2 (Fig. 5), como medida para a mitigação do impacto.

A possibilidade de utilização do SIG recém implantado no gerenciamento ambiental da Baía do Almirantado cresce à medida que surge a necessidade de escolha de áreas aptas à definição de caminhos na península Keller e no restante da AAEG, bem como de avaliação do impacto ambiental causado pelas atividades humanas na área. Para isso, é necessária a integração de novos planos de informação (e.g., delimitação dos principais campos de musgos, colônias de aves e mamíferos, indicadores químicos de poluição etc.) e estruturação de um banco de dados que possibilite a integração de dados não-espaciais derivados de estudos de avaliação ambiental realizados na área.

$\mathrm{Na}$ Antártica, o SIG serve como importante ferramenta de apoio ao gerenciamento de áreas especialmente protegidas e gerenciadas, podendo ser utilizado no planejamento de atividades científicas e logísticas, monitoramento e avaliações de impacto ambiental.

\section{Agradecimentos}

Os autores agradecem ao Environmental Systems Research Institute (ESRI) pelas licenças dos softwares, ao dr. Matthias Braun pela disponibilização de dados da IRG e a dois revisores anônimos pelos comentários construtivos. Este trabalho não teria sido realizado sem o apoio logístico da Marinha do Brasil. Arigony e Simões agradecem o Conselho Nacional de Desenvolvimento Científico e Tecnológico (CNPq) por bolsas individuais. 
ARIGONY, J.; SIMÕES, J. C.; BREMER, U. F.; DANI, N. (2002) Perspectives for environmental management of the Admiralty Bay, King George Island, Antarctica. Revista do Departamento de Geografia, n. 15, p. 91-99.

\begin{abstract}
This work describes the initial use of a geographical information system (GIS) for the Admiralty Bay Antarctic Specially Managed Area (ASMA), exemplified by preliminary results. The ASMA limits were revised as recommended by the Environmental Protection Protocol to the Antarctic Treaty (Madrid Protocol); the re-calculated area is $362 \mathrm{~km}^{2}$ (formerly $370 \mathrm{~km}^{2}$ ). A brief environmental assessment was carried out in Keller Peninsula, in the northern part of Admiralty Bay, where human activity overlaps with some breeding areas. A small or transitory environmental impact, as defined by the Madrid Protocol, was identified during the fieldwork. The need for this new GIS covering the Admiralty Bay ASMA has been growing together with the expansion of the human presence in the area and the respective environmental impact assessment.
\end{abstract}

Key words: Antarctica, King George Island, Environmental management, GIS.

Recebido em 30 de setembro de 2003, aceito em 17 de novembro de 2003.

\section{Referências bibliográficas}

ARIGONY NETO, J.; SIMÕES, J. C.; BREMER, U. F. (no prelo) Implementation of the Admiralty Bay GIS, King George Island (Antarctica). Pesquisa Antártica Brasileira. Rio de Janeiro, Academia Brasileira de Ciências.

ARIGONY NETO, J.; SIMÕES, J. C.; BREMER, U. F.; AQUINO, F. E. (2001) Detecção de mudanças ambientais na Antártica através de técnicas de sensoriamento remoto e SIG. Pesquisas em Geociências, v.28, n.2, p.337-342.

ATCPs. Antarctic Treaty Consultative Parties (1993) Protocol on environmental protection to the Antarctic Treaty, with annexes. Polar Record: SCAR Bulletin 110 - July 1993, v.29, n.170, p. 256-275.

ATCPs. Antarctic Treaty Consultative Parties (1996) A proposal prepared by Brazil and Poland, in co-ordination with Ecuador and Peru, that Admiralty Bay, King George Island (South Shetland Island) be designated as an Antarctic Specially Managed Area (ASMA): Twentieth Antarctic Treaty Consultative Meeting, Utrecht, Netherlands, 29 Abril-10 Maio de 1996.
BAS. British Antarctic Survey (2000) Antarctic digital database, Version 3.0, Manual and bibliography. Cambridge, Scientific Committee on Antarctic Research. $74 \mathrm{p}$.

BRASIL. Ministério do Meio Ambiente. Resoluções do CONAMA (Conselho Nacional do Meio Ambiente). Disponivel em: $<$ http://www.mma.gov.br>. Acesso em 15 de jan. 2003.

BRAUN, M.; SIMÕES, J. C.; VOGT, S.; BREMER, U. F.; BLINDOW, N.; PFENDER, M.; SAURER, H.; AQUINO, F. E.; FERRON, F. A. (2001) The compilation of a DTM and a new satellite image map for King George Island (Antarctica). GeoSpatial Information Science, v.4, n.2, p.47-51.

BREMER, U. F. (1998) Morfologia e bacias de drenagem da cobertura de gelo da llha Rei George, Antártica. Porto Alegre, 1 v. Dissertação (Mestrado) - Centro Estadual de Pesquisas em Sensoriamento Remoto e Meteorologia, Universidade Federal do Rio Grande do Sul.

FOREIGN \& COMMONWEALTH OFFICE (1997) List of Protected Areas in Antarctica. Londres In collaboration with the British Antarctic Survey. 
GREENPEACE (1990) Expedition Report, Greenpeace Antarctic Expedition 1989-90. Amsterdam, Stichting Greenpeace Council. 54 p.

GSSG. Geoscience Standing Scientific Group. Composite Gazetteer of Antarctica. Disponivel em: <http:// www.pnra.it/SCAR_GAZE $>$. Acesso em 15 de jan. 2003.

HANSOM, J. D.; GORDON, J. E. (1998) Antarctic environments and resources: a geographical perspective. New York, Addison Wesley Longman. $402 \mathrm{p}$.

HARRIS, C. M. (1991) Environmental effects of human activities on King George Island, South Shetland Islands, Antarctica. Polar Record, v.27, n.162, p.193-204.

IAATO. International Association of Antarctic Tour Operators. Tourism Statistics. Disponivel em: <http:// www.iaato.org.2002>. Acesso em 15 de jan. 2003.

MAGELLAN CORPORATION. User guide for the Magellan GPS ProMARK $X$ and the Magellan GPS ProMARK X-CM. Disponivel em <ftp://ftp.ashtech.com/Reference\%20Manuals>. Acesso em 15 de jan. 2003.
PROANTAR. Programa Antártico Brasileiro. Grupo de Avaliação Ambiental (GAAm). Disponivel em: $<$ http:www.secirm.mar.mil.br/inindex.htm>. Acesso em 15 de jan. 2003.

RAKUSA-SUSZCZEWSKI, S.; MIETUS, M.; PIASECKI, J. (1993) Weather and climate. In: RAKUSA-SUSZCZEWSKI, S. (ed.) The maritime Antarctic coastal ecosystem of Admiralty Bay. Warsaw, Polskiej Akademii Nauk, p.19-25.

SIMÕES, J. C.; BREMER, U. F.; AQUINO, F. A.; FERRON, F. A (1999) Morphology and variations of glacial drainage basins in the King George Island ice field, Antarctica. Annals of Glaciology, v. 29, p.220-224.

SIMÕES, J. C.; FERRON, F. A.; BRAUN, M.; ARIGONY NETO, J.; AQUINO, F. E (2001) A GIS for the Antarctic Specially Managed Area (ASMA) of Admiralty Bay, King George Island, Antarctica. Geo-spatial Information Science, v. 4, n. 2, p 8-14. 